\title{
Dynamic Opial diamond- $\alpha$ integral inequalities involving the power of a function
}

Tatjana Z Mirković

\section{"Correspondence:}

tatjana.mirkovic@visokaskola.edu.rs College of Applied Professional Studies, Filipa Filipovića 20, Vranje, 17000, Serbia

\begin{abstract}
In this paper, we present some new dynamic Opial-type diamond alpha inequalities on time scales. The obtained results are related to the function $f^{k}$.
\end{abstract}

MSC: 34N05; 26D10

Keywords: Opial-type inequality; time scale

\section{Introduction}

A time scale $\mathbb{T}$ is an arbitrary nonempty closed subset of real numbers. For $t \in \mathbb{T}$, we define the forward jump operator $\sigma: \mathbb{T} \rightarrow \mathbb{T}$ by $\sigma(t):=\inf \{s \in \mathbb{T}: s>t\}$ and the backward jump operator $\rho: \mathbb{T} \rightarrow \mathbb{T}$ by $\rho(t):=\sup \{s \in \mathbb{T}: s<t\}$. If $\sigma(t)>t$, we say that $t$ is right-scattered, whereas if $\rho(t)<t$, we say that $t$ is left-scattered. Points that are simultaneously rightscattered and left-scattered are said to be isolated. If $\sigma(t)=t$, then $t$ is called right-dense; if $\rho(t)=t$, then $t$ is called left-dense. Points that are right-dense and left-dense at the same time are called dense. The mappings $\mu, v: \mathbb{T} \rightarrow[0, \infty)$, defined by $\mu(t):=\sigma(t)-t$ and $v(t):=t-\rho(t)$, are called the forward and backward graininess function, respectively. If $\mathbb{T}$ has a left-scattered maximum $t_{1}$, then $\mathbb{T}^{k}=\mathbb{T}-\left\{t_{1}\right\}$, otherwise $\mathbb{T}^{k}=\mathbb{T}$. If $\mathbb{T}$ has a rightscattered minimum $t_{2}$, then $\mathbb{T}_{k}=\mathbb{T}-\left\{t_{2}\right\}$, otherwise $\mathbb{T}_{k}=\mathbb{T}$. Finally, $\mathbb{T}_{k}^{k}=\mathbb{T}^{k} \cap \mathbb{T}_{k}$.

Theorem 1.1 Assume $f, g: \mathbb{T} \rightarrow \mathbb{R}$ are delta differentiable at $t \in \mathbb{T}^{k}$. Then:

1. The $\operatorname{sum} f+g: \mathbb{T} \rightarrow \mathbb{R}$ is delta differentiable at $t$ with

$$
(f+g)^{\Delta}(t)=f^{\Delta}(t)+g^{\Delta}(t)
$$

2. For any constant $\alpha, \alpha f: \mathbb{T} \rightarrow \mathbb{R}$ is delta differentiable at $t$ with

$$
(\alpha f)^{\Delta}(t)=\alpha f^{\Delta}(t)
$$

3. The product $f g: \mathbb{T} \rightarrow \mathbb{R}$ is delta differentiable at $t$ with

$$
(f g)^{\Delta}(t)=f^{\Delta}(t) g(t)+f^{\sigma}(t) g^{\Delta}(t)=f(t) g^{\Delta}(t)+f^{\Delta}(t) g^{\sigma}(t) .
$$

Theorem 1.2 Assume $f, g: \mathbb{T} \rightarrow \mathbb{R}$ are nabla differentiable at $t \in \mathbb{T}_{k}$. Then:

(c) The Author(s) 2017. This article is distributed under the terms of the Creative Commons Attribution 4.0 International License (http://creativecommons.org/licenses/by/4.0/), which permits unrestricted use, distribution, and reproduction in any medium, provided you give appropriate credit to the original author(s) and the source, provide a link to the Creative Commons license, and indicate if changes were made. 
1. The sum $f+g: \mathbb{T} \rightarrow \mathbb{R}$ is nabla differentiable at $t$ with

$$
(f+g)^{\nabla}(t)=f^{\nabla}(t)+g^{\nabla}(t) .
$$

2. For any constant $\alpha, \alpha f: \mathbb{T} \rightarrow \mathbb{R}$ is nabla differentiable at $t$ with

$$
(\alpha f)^{\nabla}(t)=\alpha f^{\nabla}(t)
$$

3. The product $f g: \mathbb{T} \rightarrow \mathbb{R}$ is nabla differentiable at $t$ with

$$
(f g)^{\nabla}(t)=f^{\nabla}(t) g(t)+f^{\rho}(t) g^{\nabla}(t)=f(t) g^{\nabla}(t)+f^{\nabla}(t) g^{\rho}(t) .
$$

The following formulas will be used in our paper:

$$
\begin{aligned}
& \left(f^{l+1}\right)^{\Delta}=\left\{\sum_{k=0}^{l} f^{k}\left(f^{\sigma}\right)^{l-k}\right\} f^{\Delta}, \quad l \in N, \\
& \left(f^{l+1}\right)^{\nabla}=\left\{\sum_{k=0}^{l} f^{k}\left(f^{\rho}\right)^{l-k}\right\} f^{\nabla}, \quad l \in N .
\end{aligned}
$$

Definition 1.3 Let $0 \leq \alpha \leq 1$ and let $f$ be both delta and nabla differentiable at $t \in \mathbb{T}_{k}^{k}$. Then $f$ is diamond- $\alpha$ differentiable at $t$ and $f^{\diamond}(t)=\alpha f^{\Delta}(t)+(1-\alpha) f^{\nabla}(t)$.

Definition 1.4 Let $a, b \in \mathbb{T}, a<b, f: \mathbb{T} \rightarrow \mathbb{R}$ and $\alpha \in[0,1]$. The diamond- $\alpha$ integral of $t$ on $[a, b]_{\mathbb{T}}$ is defined by

$$
\int_{a}^{b} f(t) \diamond_{\alpha} t=\alpha \int_{a}^{b} f(t) \Delta t+(1-\alpha) \int_{a}^{b} f(t) \nabla t .
$$

Theorem 1.5 Let $f, g: \mathbb{T} \rightarrow \mathbb{R}$ be $\diamond_{\alpha}$-differentiable at $t \in \mathbb{T}$. Then

1. $f+g$ is $\diamond_{\alpha}$-differentiable $t \in \mathbb{T}$ with $(f+g)^{\diamond_{\alpha}}=f^{\diamond_{\alpha}}+g^{\diamond_{\alpha}}$,

2. $f g$ is $\diamond_{\alpha}$-differentiable at $t \in \mathbb{T}$ with $(f g)^{\diamond_{\alpha}}=f^{\diamond_{\alpha}} g+\alpha f^{\sigma} g^{\Delta}+(1-\alpha) f^{\rho} g^{\nabla}$.

Many authors have studied the theory of integral inequalities on time scales (see, for example, [1-10]). In [3], the following Opial inequality on time scales was established.

Theorem 1.6 ([3]) For a delta differentiable $f:[0, h] \cap \mathbb{T} \rightarrow \mathbb{R}$ with $f(0)=0$, we have

$$
\int_{0}^{h}\left|\left(f+f^{\sigma}\right) f^{\Delta}\right| \Delta t \leq h \int_{0}^{h}\left|f^{\Delta}\right|^{2} \Delta t
$$

with equality when $f(t)=$ ct.

In [1], the authors established the following theorem.

Theorem 1.7 ([1]) Let $\omega(t)$ be positive and continuous on $(0, h)$ with $\int_{0}^{h} \omega^{1-q} \Delta t<\infty, q>1$. For a differentiable $f:[0, h] \rightarrow \mathbb{R}$ with $f(0)=0$, we have

$$
\int_{0}^{h}\left|\left(f+f^{\sigma}\right) f^{\Delta}\right| \Delta t \leq\left(\int_{0}^{h} \omega^{1-q} \Delta t\right)^{\frac{2}{q}}\left(\int_{0}^{h} \omega\left|f^{\Delta}\right|^{p} \Delta t\right)^{\frac{2}{p}},
$$

where $p>1$ and $\frac{1}{p}+\frac{1}{q}=1$, and with equality when $f(t)=c \int_{0}^{t} \omega^{1-q} \Delta \tau$ for a constant $c$. 


\section{Main results}

In this section, we present our results.

Theorem 2.1 Let $T$ be a time scale. For $\diamond_{\alpha}$ differentiable $f:[0, h] \cap T \rightarrow R$, with $f(0)=0$ we have

$$
\int_{0}^{h}\left|f^{k}\right|^{\diamond_{\alpha}}(t) \diamond_{\alpha} t \leq h^{k-1} \int_{0}^{h}\left|f^{\diamond_{\alpha}}\right|^{k}(t) \diamond_{\alpha} t .
$$

Proof Starting with the left side of (2), we obtain

$$
\begin{aligned}
\int_{0}^{h}\left|f^{k}\right|^{\diamond_{\alpha}}(t) \diamond_{\alpha}(t)= & \int_{0}^{h}\left|f \cdot f^{k-1}\right|^{\diamond_{\alpha}}(t) \diamond_{\alpha}(t) \\
= & \int_{0}^{h}\left|f^{k-1} f^{\diamond_{\alpha}}+\alpha f^{\sigma}\left(f^{k-1}\right)^{\Delta}+(1-\alpha) f^{\rho}\left(f^{k-1}\right)^{\nabla}\right|(t) \diamond_{\alpha}(t) \\
= & \alpha \int_{0}^{h}\left|f^{k-1} f^{\diamond_{\alpha}}+\alpha f^{\sigma}\left(f^{k-1}\right)^{\Delta}+(1-\alpha) f^{\rho}\left(f^{k-1}\right)^{\nabla}\right|(t) \Delta t \\
& +(1-\alpha) \int_{0}^{h}\left|f^{k-1} f^{\diamond_{\alpha}}+\alpha f^{\sigma}\left(f^{k-1}\right)^{\Delta}+(1-\alpha) f^{\rho}\left(f^{k-1}\right)^{\nabla}\right|(t) \nabla t \\
\leq & \alpha \int_{0}^{h}\left|f^{k-1} f^{\diamond_{\alpha}}\right|(t) \Delta t+\alpha^{2} \int_{0}^{h}\left|f^{\sigma}\left(f^{k-1}\right)^{\Delta}\right|(t) \Delta t \\
& +\alpha(1-\alpha) \int_{0}^{h}\left|f^{\rho}\left(f^{k-1}\right)^{\nabla}\right|(t) \Delta t+(1-\alpha) \int_{0}^{h}\left|f^{k-1} f^{\diamond_{\alpha}}\right|(t) \nabla t \\
& +\alpha(1-\alpha) \int_{0}^{h}\left|f^{\sigma}\left(f^{k-1}\right)^{\Delta}\right|(t) \nabla t+(1-\alpha)^{2} \int_{0}^{h}\left|f^{\rho}\left(f^{k-1}\right)^{\nabla}\right|(t) \nabla t .
\end{aligned}
$$

Using Definition 1.3, we get

$$
\begin{aligned}
\int_{0}^{h}\left|f^{k}\right|^{\diamond_{\alpha}}(t) \diamond_{\alpha}(t) \leq & \alpha \int_{0}^{h}\left|\alpha f^{k-1} f^{\Delta}+(1-\alpha) f^{k-1} f^{\nabla}\right|(t) \Delta t \\
& +\alpha^{2} \int_{0}^{h}\left|f^{\sigma}\left(f^{k-1}\right)^{\Delta}\right|(t) \Delta t+\alpha(1-\alpha) \int_{0}^{h}\left|f^{\rho}\left(f^{k-1}\right)^{\nabla}\right|(t) \Delta t \\
& +(1-\alpha) \int_{0}^{h}\left|\alpha f^{k-1} f^{\Delta}+(1-\alpha) f^{k-1} f^{\nabla}\right|(t) \nabla t \\
& +\alpha(1-\alpha) \int_{0}^{h}\left|f^{\sigma}\left(f^{k-1}\right)^{\Delta}\right|(t) \nabla t+(1-\alpha)^{2} \int_{0}^{h}\left|f^{\rho}\left(f^{k-1}\right)^{\nabla}\right|(t) \nabla t \\
\leq & \alpha^{2} \int_{0}^{h}\left|f^{k-1} f^{\Delta}\right|(t) \Delta t+\alpha(1-\alpha) \int_{0}^{h}\left|f^{k-1} f^{\nabla}\right|(t) \Delta t \\
& +\alpha^{2} \int_{0}^{h}\left|f^{\sigma}\left(f^{k-1}\right)^{\Delta}\right|(t) \Delta t+\alpha(1-\alpha) \int_{0}^{h}\left|f^{\rho}\left(f^{k-1}\right)^{\nabla}\right|(t) \Delta t \\
& +\alpha(1-\alpha) \int_{0}^{h}\left|f^{k-1} f^{\Delta}\right|(t) \nabla t+(1-\alpha)^{2} \int_{0}^{h}\left|f^{k-1} f^{\nabla}\right|(t) \nabla t \\
& +\alpha(1-\alpha) \int_{0}^{h}\left|f^{\sigma}\left(f^{k-1}\right)^{\Delta}\right|(t) \nabla t+(1-\alpha)^{2} \int_{0}^{h}\left|f^{\rho}\left(f^{k-1}\right)^{\nabla}\right|(t) \nabla t .
\end{aligned}
$$


We find that

$$
\begin{aligned}
\int_{0}^{h}\left|f^{\sigma}\left(f^{k-1}\right)^{\Delta}\right|(t) \Delta t= & \int_{0}^{h}\left|f^{\sigma}\left(f \cdot f^{k-2}\right)^{\Delta}\right|(t) \Delta t \\
= & \int_{0}^{h}\left|f^{\sigma}\left(f^{\Delta} f^{k-2}+f^{\sigma}\left(f \cdot f^{k-3}\right)^{\Delta}\right)\right|(t) \Delta t \\
& \vdots \\
& =\int_{0}^{h}\left|f^{\sigma}\left(f^{\Delta} f^{k-2}+f^{\sigma} f^{\Delta} f^{k-3}+\cdots+\left(f^{\sigma}\right)^{2} f^{\Delta}\right)\right|(t) \Delta t \\
& =\int_{0}^{h}\left|f^{\sigma} f^{k-2}+\left(f^{\sigma}\right)^{2} f^{k-3}+\cdots+\left(f^{\sigma}\right)\right|\left|f^{\Delta}\right|(t) \Delta t \\
& =\int_{0}^{h}\left|\sum_{n=0}^{k-2} f^{n}\left(f^{\sigma}\right)^{k-1-n}\right|\left|f^{\Delta}\right|(t) \Delta t .
\end{aligned}
$$

Similarly,

$$
\int_{0}^{h}\left|f^{\rho}\left(f^{k-1}\right)^{\nabla}\right|(t) \Delta t=\int_{0}^{h}\left|\sum_{n=0}^{k-2} f^{n}\left(f^{\rho}\right)^{k-1-n}\right|\left|f^{\nabla}\right|(t) \nabla t .
$$

Therefore,

$$
\begin{aligned}
\int_{0}^{h}\left|f^{k}\right|^{\diamond_{\alpha}}(t) \diamond_{\alpha} t \leq & \alpha^{2} \int_{0}^{h}\left|f^{k-1} f^{\Delta}\right|(t) \Delta t+\alpha(1-\alpha) \int_{0}^{h}\left|f^{k-1} f^{\nabla}\right|(t) \Delta t \\
& +\alpha^{2} \int_{0}^{h}\left|\sum_{n=0}^{k-2} f^{n}\left(f^{\sigma}\right)^{k-1-n}\right|\left|f^{\Delta}\right|(t) \Delta t \\
& +\alpha(1-\alpha) \int_{0}^{h}\left|\sum_{n=0}^{k-2} f^{n}\left(f^{\rho}\right)^{k-1-n}\right|\left|f^{\nabla}\right|(t) \Delta t \\
& +\alpha(1-\alpha) \int_{0}^{h}\left|f^{k-1} f^{\Delta}\right|(t) \nabla t+(1-\alpha)^{2} \int_{0}^{h}\left|f^{k-1} f^{\nabla}\right|(t) \nabla t \\
& +\alpha(1-\alpha) \int_{0}^{h}\left|\sum_{n=0}^{k-2} f^{n}\left(f^{\sigma}\right)^{k-1-n}\right|\left|f^{\Delta}\right| \nabla t \\
& +(1-\alpha)^{2} \int_{0}^{h}\left|\sum_{n=0}^{k-2} f^{n}\left(f^{\rho}\right)^{k-1-n}\right|\left|f^{\nabla}\right|(t) \nabla t \\
= & \alpha^{2} \int_{0}^{h}\left(\left|f^{k-1}\right|+\left|\sum_{n=0}^{k-2} f^{n}\left(f^{\sigma}\right)^{k-1-n}\right|\right)\left|f^{\Delta}(t)\right| \Delta t \\
& +\alpha(1-\alpha) \int_{0}^{h}\left(\left|f^{k-1}\right|+\left|\sum_{n=0}^{k-2} f^{n}\left(f^{\rho}\right)^{k-1-n}\right|\right)\left|f^{\nabla}(t)\right| \Delta t \\
& +\alpha(1-\alpha) \int_{0}^{h}\left(\left|f^{k-1}\right|+\left|\sum_{n=0}^{k-2} f^{n}\left(f^{\sigma}\right)^{k-1-n}\right|\right)\left|f^{\Delta}(t)\right| \nabla t
\end{aligned}
$$




$$
\begin{aligned}
& +(1-\alpha)^{2} \int_{0}^{h}\left(\left|f^{k-1}\right|+\left|\sum_{n=0}^{k-2} f^{n}\left(f^{\rho}\right)^{k-1-n}\right|\right)\left|f^{\nabla}(t)\right| \nabla t \\
\leq & \alpha^{2} \int_{0}^{h}\left(\left|f^{k-1}\right|+\sum_{n=0}^{k-2}\left|f^{n}\left(f^{\sigma}\right)^{k-1-n}\right|\right)\left|f^{\Delta}(t)\right| \Delta t \\
& +\alpha(1-\alpha) \int_{0}^{h}\left(\left|f^{k-1}\right|+\sum_{n=0}^{k-2}\left|f^{n}\left(f^{\rho}\right)^{k-1-n}\right|\right)\left|f^{\nabla}(t)\right| \Delta t \\
& +\alpha(1-\alpha) \int_{0}^{h}\left(\left|f^{k-1}\right|+\sum_{n=0}^{k-2}\left|f^{n}\left(f^{\sigma}\right)^{k-1-n}\right|\right)\left|f^{\Delta}(t)\right| \nabla t \\
& +(1-\alpha)^{2} \int_{0}^{h}\left(\left|f^{k-1}\right|+\sum_{n=0}^{k-2}\left|f^{n}\left(f^{\rho}\right)^{k-1-n}\right|\right)\left|f^{\nabla}(t)\right| \nabla t \\
= & \alpha^{2} \int_{0}^{h}\left(\sum_{n=0}^{k-1}\left|f^{n}\right|\left|\left(f^{\sigma}\right)^{k-1-n}\right|\right)\left|f^{\Delta}(t)\right| \Delta t \\
& +\alpha(1-\alpha) \int_{0}^{h}\left(\sum_{n=0}^{k-1}\left|f^{n}\right|\left|\left(f^{\rho}\right)^{k-1-n}\right|\right)\left|f^{\nabla}(t)\right| \Delta t \\
& +\alpha(1-\alpha) \int_{0}^{h}\left(\sum_{n=0}^{k-1}\left|f^{n}\right|\left|\left(f^{\sigma}\right)^{k-1-n}\right|\right)\left|f^{\Delta}(t)\right| \nabla t \\
& +(1-\alpha)^{2} \int_{0}^{h}\left(\sum_{n=0}^{k-1}\left|f^{n}\right|\left|\left(f^{\rho}\right)^{k-1-n}\right|\right)\left|f^{\nabla}(t)\right| \nabla t .
\end{aligned}
$$

Consider $g(t)=\int_{0}^{t}\left|f^{\diamond_{\alpha}}(s)\right| \diamond_{\alpha} s$. Then we have $g^{\Delta}(t)=\left|f^{\Delta}(t)\right|, g^{\nabla}(t)=\left|f^{\nabla}(t)\right|$, and $|f| \leq g$, so that $g(t)=\int_{0}^{t}\left|f^{\diamond_{\alpha}}(s)\right| \diamond_{\alpha} s \geq\left|\int_{0}^{t} f^{\diamond_{\alpha}}(s) \diamond_{\alpha} s\right|=|f(t)-f(0)|=|f(t)|$.

The above inequality becomes

$$
\begin{aligned}
\int_{0}^{h}\left|f^{k}\right|^{\diamond_{\alpha}}(t) \diamond_{\alpha} \leq & \alpha^{2} \int_{0}^{h}\left(\sum_{n=0}^{k-1} g^{n}\left(g^{\sigma}\right)^{k-1-n}\right)\left(g^{\Delta}\right)(t) \Delta t \\
& +\alpha(1-\alpha) \int_{0}^{h}\left(\sum_{n=0}^{k-1} g^{n}\left(g^{\rho}\right)^{k-1-n}\right)\left(g^{\nabla}\right)(t) \Delta t \\
& +\alpha(1-\alpha) \int_{0}^{h}\left(\sum_{n=0}^{k-1} g^{n}\left(g^{\sigma}\right)^{k-1-n}\right)\left(g^{\Delta}\right)(t) \nabla t \\
& +(1-\alpha)^{2} \int_{0}^{h}\left(\sum_{n=0}^{k-1} g^{n}\left(g^{\rho}\right)^{k-1-n}\right)\left(g^{\nabla}\right)(t) \nabla t \\
= & \alpha^{2} \int_{0}^{h}\left(g^{k}\right)^{\Delta}(t) \Delta t+\alpha(1-\alpha) \int_{0}^{h}\left(g^{k}\right)^{\nabla}(t) \Delta t \\
& +\alpha(1-\alpha) \int_{0}^{h}\left(g^{k}\right)^{\Delta}(t) \nabla t+(1-\alpha)^{2} \int_{0}^{h}\left(g^{k}\right)^{\nabla}(t) \nabla t \\
= & \alpha\left[\alpha \int_{0}^{h}\left(g^{k}\right)^{\Delta} \Delta t+(1-\alpha) \int_{0}^{h}\left(g^{k}\right)^{\Delta} \nabla t\right]
\end{aligned}
$$


Mirković Journal of Inequalities and Applications （2017） 2017:139

Page 6 of 10

$$
\begin{aligned}
& +(1-\alpha)\left[\int_{0}^{h} \alpha\left(g^{k}\right)^{\nabla} \Delta t+(1-\alpha) \int_{0}^{h}\left(g^{k}\right)^{\nabla} \nabla t\right] \\
= & \alpha \int_{0}^{h}\left(g^{k}\right)^{\Delta} \diamond_{\alpha}+(1-\alpha) \int_{0}^{h}\left(g^{k}\right)^{\nabla} \diamond_{\alpha}=\int_{0}^{h}\left(g^{k}\right)(t)^{\diamond_{\alpha}} \diamond_{\alpha} \\
= & \left.g^{k}(t)\right|_{0} ^{h}=g^{k}(h)-g^{k}(0)=[g(h)]^{k}=\left[\int_{0}^{h}\left|f^{\diamond_{\alpha}}(s)\right| \diamond_{\alpha} s\right]^{k} .
\end{aligned}
$$

By using Hölder's inequality with indices $p=\frac{k}{k-1}$ and $q=k$, we obtain

$$
\begin{aligned}
{\left[\int_{0}^{h} 1 \cdot\left|f^{\diamond_{\alpha}}(s)\right| \diamond_{\alpha} s\right]^{k} } & \leq\left[\left(\int_{0}^{h} 1 \frac{k}{k-1} \diamond_{\alpha} s\right)^{\frac{k-1}{k}}\left(\int_{0}^{h}\left|f^{\diamond_{\alpha}}(s)\right|^{k} \diamond_{\alpha} s\right)^{\frac{1}{k}}\right]^{k} \\
& =\left(\int_{0}^{h} \diamond_{\alpha} s\right)^{k-1}\left(\int_{0}^{h}\left|f^{\diamond_{\alpha}}(s)\right|^{k} \diamond_{\alpha} s\right) \\
& =\left(\left.s\right|_{0} ^{h}\right)^{k-1} \int_{0}^{h}\left|f^{\diamond_{\alpha}}(s)\right|^{k} \diamond_{\alpha} s \\
& =h^{k-1} \int_{0}^{h}\left|f^{\diamond_{\alpha}}(s)\right|^{k} \diamond_{\alpha} s,
\end{aligned}
$$

hence the proof is complete.

Theorem 2.2 Let $\omega(t)$ be positive and continuous on $(0, h)$, with $\int_{0}^{h} \omega^{1-q}(t) \Delta t<\infty, q>1$. For differentiable $f:[0, h] \rightarrow \mathbb{R}$ with $f(0)=0$ we have

$$
\int_{0}^{h}\left|f^{k}\right|^{\Delta} \Delta t \leq\left(\int_{0}^{h} \omega^{1-q} \Delta t\right)^{\frac{k}{q}}\left(\int_{0}^{h} \omega\left|f^{\Delta}\right|^{p} \Delta t\right)^{\frac{k}{q}},
$$

where $p>1$ and $\frac{1}{p}+\frac{1}{q}=1$.

Proof We take $g(t)=\int_{0}^{t}\left|f^{\Delta}(s)\right| \Delta s$. Then $|f(t)| \leq g(t), g^{\Delta}(t)=\left|f^{\Delta}(t)\right|$, so we have

$$
\begin{aligned}
\int_{0}^{h}\left|f^{k}\right|^{\Delta} \Delta t & =\int_{0}^{h}\left|\sum_{k=0}^{n-1} f^{k}\left(f^{\sigma}\right)^{n-1-k}\right|\left|f^{\Delta}\right|(t) \Delta t \\
& \leq \int_{0}^{h}\left(\sum_{k=0}^{n-1} g^{k}\left(g^{\sigma}\right)^{n-1-k}\right)\left(g^{\Delta}\right)(t) \Delta t=\int_{0}^{h}\left(g^{k}\right)^{\Delta} \Delta t \\
& =g^{k}(h)-g^{k}(0)=g^{k}(h)=\left(\int_{0}^{h}\left|f^{\Delta}\right|(t) \Delta t\right)^{k} \\
& =\left(\int_{0}^{h} \omega^{-\frac{1}{p}} \omega^{\frac{1}{p}}\left|f^{\Delta}\right|(t) \Delta t\right)^{k} \\
& \leq\left[\left(\int_{0}^{h}\left(\omega^{-\frac{1}{p}}\right)^{q} \Delta t\right)^{\frac{1}{q}}\left(\int_{0}^{h}\left(\omega^{\frac{1}{p}}\left|f^{\Delta}\right|\right)^{p}(t) \Delta t\right)^{\frac{1}{p}}\right]^{k} \\
& =\left(\int_{0}^{h} \omega^{1-q} \Delta t\right)^{\frac{k}{q}}\left(\int_{0}^{h}\left(\omega\left|f^{\Delta}\right|\right)^{p}(t) \Delta t\right)^{\frac{k}{p}} .
\end{aligned}
$$


Theorem 2.3 Let $\omega(t)$ be positive and continuous on $(0, h)$, with $\int_{0}^{h} \omega^{1-q}(t) \nabla t<\infty, q>1$. For differentiable $f:[0, h] \rightarrow \mathbb{R}$ with $f(0)=0$ we have

$$
\int_{0}^{h}\left|f^{k}\right|^{\nabla} \nabla t \leq\left(\int_{0}^{h} \omega^{1-q} \nabla t\right)^{\frac{k}{q}}\left(\int_{0}^{h} \omega\left|f^{\nabla}\right|^{p} \nabla t\right)^{\frac{k}{q}},
$$

where $p>1$ and $\frac{1}{p}+\frac{1}{q}=1$.

Theorem 2.4 Assume that $p>1, q=\frac{p}{p-1}, \alpha \in[0,1], h \in(0, \infty)_{\mathbb{T}}, \omega \in \mathbb{C}\left([0, h]_{\mathbb{T}},(0, \infty)\right)$ and $f \in \mathbb{C}_{\diamond_{\alpha}}^{1}\left([0, h]_{\mathbb{T}}, \mathbb{R}\right)$. If $\alpha f^{\Delta} \geq 0,(1-\alpha) f^{\nabla} \geq 0$ and $f(0)=0$ then

$$
\begin{aligned}
& \alpha^{k} \int_{0}^{h}\left|\left(f^{k}\right)^{\Delta}(t)\right| \Delta t+(1-\alpha)^{k} \int_{0}^{h}\left|\left(f^{k}\right)^{\nabla}(t)\right| \nabla t \\
& \leq\left(\int_{0}^{h} \omega^{1-q}(t) \diamond_{\alpha} t\right)^{\frac{k}{q}}\left(\int_{0}^{h} \omega(t)\left|f^{\diamond_{\alpha}}(t)\right|^{p} \diamond_{\alpha} t\right)^{\frac{k}{p}} .
\end{aligned}
$$

Proof By Theorems 2.2, 2.3, Hölder's inequality and $k=\frac{k}{q}+(1+p) \frac{k}{p}$, we get

$$
\begin{aligned}
& \alpha^{k} \int_{0}^{h}\left|\left(f^{k}\right)^{\Delta}(t)\right| \Delta t+(1-\alpha)^{k} \int_{0}^{h}\left|\left(f^{k}\right)^{\nabla}(t)\right| \nabla t \\
& =\alpha^{\frac{k}{q}+(1+p) \frac{k}{p}} \int_{0}^{h}\left|\left(f^{k}\right)^{\Delta}(t)\right| \Delta t+(1-\alpha)^{\frac{k}{q}+(1+p) \frac{k}{p}} \int_{0}^{h}\left|\left(f^{k}\right)^{\nabla}(t)\right| \nabla t \\
& \leq \alpha^{\frac{k}{q}+(1+p) \frac{k}{p}}\left(\int_{0}^{h} \omega^{1-q}(t) \Delta t\right)^{\frac{k}{q}}\left(\int_{0}^{h} \omega(t)\left|f^{\Delta}(t)\right|^{p} \Delta t\right)^{\frac{k}{p}} \\
& +(1-\alpha)^{\frac{k}{q}+(1+p) \frac{k}{p}}\left(\int_{0}^{h} \omega^{1-q}(t) \nabla t\right)^{\frac{k}{q}}\left(\int_{0}^{h} \omega(t)\left|f^{\nabla}(t)\right|^{p} \nabla t\right)^{\frac{k}{p}} \\
& \leq\left(\alpha \int_{0}^{h} \omega^{1-q}(t) \Delta t\right)^{\frac{k}{q}}\left(\alpha \int_{0}^{h} \omega(t)\left|\alpha f^{\Delta}(t)+(1-\alpha) f^{\nabla}(t)\right|^{p} \Delta t\right)^{\frac{k}{p}} \\
& +\left((1-\alpha) \int_{0}^{h} \omega^{1-q}(t) \nabla t\right)^{\frac{k}{q}} \\
& \cdot\left((1-\alpha) \int_{0}^{h} \omega(t)\left|\alpha f^{\Delta}(t)+(1-\alpha) f^{\nabla}(t)\right|^{p} \nabla t\right)^{\frac{k}{p}} \\
& =\left(\alpha \int_{0}^{h} \omega^{1-q}(t) \Delta t\right)^{\frac{k}{q}}\left(\alpha \int_{0}^{h} \omega(t)\left|f^{\diamond_{\alpha}}(t)\right|^{p} \Delta t\right)^{\frac{k}{p}} \\
& +\left((1-\alpha) \int_{0}^{h} \omega^{1-q}(t) \nabla t\right)^{\frac{k}{q}}\left((1-\alpha) \int_{0}^{h} \omega(t)\left|f^{\diamond \alpha}(t)\right|^{p} \nabla t\right)^{\frac{k}{p}} \\
& \leq\left[\left(\alpha \int_{0}^{h} \omega^{1-q}(t) \Delta t\right)^{k}+\left((1-\alpha) \int_{0}^{h} \omega^{1-q}(t) \nabla t\right)^{k}\right]^{\frac{1}{q}} \\
& \cdot\left[\left(\alpha \int_{0}^{h} \omega(t)\left|f^{\diamond_{\alpha}}(t)\right|^{p} \Delta t\right)^{k}+\left((1-\alpha) \int_{0}^{h} \omega(t)\left|f^{\diamond \alpha}(t)\right|^{p} \nabla t\right)^{k}\right]^{\frac{1}{q}} \\
& \leq\left(\alpha \int_{0}^{h} \omega^{1-q}(t) \Delta t+(1-\alpha) \int_{0}^{h} \omega^{1-q}(t) \nabla t\right)^{\frac{k}{q}}
\end{aligned}
$$




$$
\begin{aligned}
& \cdot\left(\alpha \int_{0}^{h} \omega(t)\left|f^{\diamond_{\alpha}}(t)\right|^{p} \Delta t+(1-\alpha) \int_{0}^{h} \omega(t)\left|f^{\diamond_{\alpha}}(t)\right|^{p} \nabla t\right)^{\frac{k}{p}} \\
= & \left(\int_{0}^{h} \omega^{1-q}(t) \diamond_{\alpha} t\right)^{\frac{k}{q}}\left(\int_{0}^{h} \omega(t)\left|f^{\diamond_{\alpha}}(t)\right|^{p} \diamond_{\alpha} t\right)^{\frac{k}{p}} .
\end{aligned}
$$

Theorem 2.5 Assume that $1<p \leq 2, q=\frac{p}{p-1}, \alpha \in[0,1], h \in(0, \infty)_{\mathbb{T}}, \omega \in \mathbb{C}\left([0, h]_{\mathbb{T}},(0, \infty)\right)$ and $f \in \mathbb{C}_{\diamond_{\alpha}}^{1}\left([0, h]_{\mathbb{T}}, \mathbb{R}\right)$. If $\alpha f^{\Delta} \geq 0,(1-\alpha) f^{\nabla} \geq 0$ and $f(0)=0$, then

$$
\begin{aligned}
& \alpha^{k} \int_{0}^{u}\left|\left(f^{k}\right)^{\Delta}(t)\right| \Delta t+(1-\alpha)^{k} \int_{0}^{u}\left|\left(f^{k}\right)^{\nabla}(t)\right| \nabla t \\
& \leq \sum_{j=0}^{k-2} \alpha^{j}\left(\begin{array}{l}
k \\
j
\end{array}\right) \gamma^{j} \beta^{\frac{k-j}{q}}\left[\int_{0}^{h} \omega(t)\left|g^{\diamond_{\alpha}}(t)\right|^{p} \diamond_{\alpha} t\right]^{\frac{k-1}{p}} \\
& +\alpha^{k-1}\left(\begin{array}{c}
k \\
k-1
\end{array}\right) \gamma^{k-1}(f(h)-f(0)),
\end{aligned}
$$

where $\beta:=\min _{u \in[0, h]_{T}} v(u), v(u)=\max \left\{\int_{0}^{u} \omega^{1-q}(t) \diamond_{\alpha} t, \int_{u}^{h} \omega^{1-q}(t) \diamond_{\alpha} t\right\}, \gamma:=\max \{|f(0)|$, $|f(h)|\}$.

Proof We let $u \in[0, h]_{\mathbb{T}}$ be arbitrary. By applying Theorem 2.4 to the function $g(t)=f(t)-$ $f(0)$, we obtain

$$
\begin{aligned}
& \alpha^{k} \int_{0}^{u}\left|\left(f^{k}\right)^{\Delta}(t)\right| \Delta t+(1-\alpha)^{k} \int_{0}^{u}\left|\left(f^{k}\right)^{\nabla}(t)\right| \nabla t \\
& =\alpha^{k} \int_{0}^{u}\left|\sum_{j=0}^{k-1}\left(\begin{array}{l}
k \\
j
\end{array}\right)\left(g^{k-j}\right)^{\Delta} f^{j}(0)\right| \Delta t \\
& +(1-\alpha)^{k} \int_{0}^{u}\left|\sum_{j=0}^{k-1}\left(\begin{array}{l}
k \\
j
\end{array}\right)\left(g^{k-j}\right)^{\nabla} f^{j}(0)\right| \nabla t \\
& \leq\left(\begin{array}{l}
k \\
0
\end{array}\right)\left[\alpha^{k} \int_{0}^{u}\left|g^{k}\right|^{\Delta} \Delta t+(1-\alpha)^{k} \int_{0}^{u}\left|g^{k}\right|^{\nabla} \nabla t\right] \\
& +\alpha\left(\begin{array}{l}
k \\
1
\end{array}\right)|f(0)|\left[\alpha^{k-1} \int_{0}^{u}\left|g^{k-1}\right|^{\Delta} \Delta t+(1-\alpha)^{k-1} \int_{0}^{u}\left|g^{k-1}\right|^{\nabla} \nabla t\right] \\
& +\alpha^{2}\left(\begin{array}{l}
k \\
2
\end{array}\right)\left|f^{2}(0)\right|\left[\alpha^{k-2} \int_{0}^{u}\left|g^{k-2}\right|^{\Delta} \Delta t+(1-\alpha)^{k-2} \int_{0}^{u}\left|g^{k-2}\right|^{\nabla} \nabla t\right] \\
& \vdots \\
& +\alpha^{k-2}\left(\begin{array}{c}
k \\
k-2
\end{array}\right)\left|f^{k-2}(0)\right|\left[\alpha^{2} \int_{0}^{u}\left|g^{2}\right|^{\Delta} \Delta t+(1-\alpha)^{2} \int_{0}^{u}\left|g^{2}\right|^{\nabla} \nabla t\right] \\
& +\alpha^{k-1}\left(\begin{array}{c}
k \\
k-1
\end{array}\right)\left|f^{k-1}(0)\right|\left[\alpha \int_{0}^{u}|f|^{\Delta} \Delta t+(1-\alpha) \int_{0}^{u}|f|^{\nabla} \nabla t\right] \\
& \leq\left(\begin{array}{l}
k \\
0
\end{array}\right)\left[\int_{0}^{u} \omega^{1-q}(t) \diamond_{\alpha} t\right]^{\frac{k}{q}}\left[\int_{0}^{u} \omega(t)\left|g^{\diamond_{\alpha}}(t)\right|^{p} \diamond_{\alpha} t\right]^{\frac{k}{p}}
\end{aligned}
$$




$$
\begin{aligned}
& +\alpha\left(\begin{array}{l}
k \\
1
\end{array}\right)|f(0)|\left[\int_{0}^{u} \omega^{1-q}(t) \diamond_{\alpha} t\right]^{\frac{k-1}{q}}\left[\int_{0}^{u} \omega(t)\left|g^{\diamond_{\alpha}}(t)\right|^{p} \diamond_{\alpha} t\right]^{\frac{k-1}{p}} \\
& +\alpha^{2}\left(\begin{array}{l}
k \\
2
\end{array}\right)\left|f^{2}(0)\right|\left[\int_{0}^{u} \omega^{1-q}(t) \diamond_{\alpha} t\right]^{\frac{k-2}{q}}\left[\int_{0}^{u} \omega(t)\left|g^{\diamond_{\alpha}}(t)\right|^{p} \diamond_{\alpha} t\right]^{\frac{k-2}{p}} \\
& \vdots \\
& +\alpha^{k-2}\left(\begin{array}{c}
k \\
k-2
\end{array}\right)\left|f^{k-2}(0)\right|\left[\int_{0}^{u} \omega^{1-q}(t) \diamond_{\alpha} t\right]^{\frac{2}{q}} \\
& +\left[\int_{0}^{u} \omega(t)\left|g^{\diamond_{\alpha}}(t)\right|^{p} \diamond_{\alpha} t\right]^{\frac{2}{p}} \\
& +\alpha^{k-1}\left(\begin{array}{c}
k \\
k-1
\end{array}\right)\left|f^{k-1}(0)\right| \alpha \int_{0}^{u}\left|f^{\Delta}(t)\right| \Delta t \\
& +\alpha^{k-1}\left(\begin{array}{c}
k \\
k-1
\end{array}\right)\left|f^{k-1}(0)\right|(1-\alpha) \int_{0}^{u}\left|f^{\nabla}(t)\right| \nabla t \\
& \sum_{j-2}^{k} \alpha^{j}\left(\begin{array}{c}
k \\
j
\end{array}\right)\left|f^{j}(0)\right|\left[\int_{0}^{u} \omega^{1-q}(t) \diamond_{\alpha} t\right]^{\frac{k-j}{q}}\left[\int_{0}^{u} \omega(t)\left|g^{\diamond_{\alpha}}(t)\right|^{p} \diamond_{\alpha} t\right]^{\frac{k-j}{p}} \\
& +\alpha^{k-1}\left(\begin{array}{c}
k \\
k-1
\end{array}\right) \gamma^{k-1}(f(u)-f(0)) .
\end{aligned}
$$

Similarly,

$$
\begin{aligned}
& \alpha^{k} \int_{u}^{h}\left|\left(f^{k}\right)^{\Delta}(t)\right| \Delta t+(1-\alpha)^{k} \int_{u}^{h}\left|\left(f^{k}\right)^{\nabla}(t)\right| \nabla t \\
& \leq \sum_{j=0}^{k-2} \alpha^{j}\left(\begin{array}{l}
k \\
j
\end{array}\right)\left|f^{j}(0)\right|\left[\int_{u}^{h} \omega^{1-q}(t) \diamond_{\alpha} t\right]^{\frac{k-j}{q}}\left[\int_{u}^{h} \omega(t)\left|g^{\diamond_{\alpha}}(t)\right|^{p} \diamond_{\alpha} t\right]^{\frac{k-j}{p}} \\
& \quad+\alpha^{k-1}\left(\begin{array}{c}
k \\
k-1
\end{array}\right) \gamma^{k-1}(f(h)-f(u)) .
\end{aligned}
$$

Adding these two inequalities and taking into account that $a^{r}+b^{r} \leq(a+b)^{r}$ holds, for $a, b \geq 0$ and $r \geq 1$, yield the desired inequality.

\section{Conclusion}

In this paper, we have obtained several Opial-type integral inequalities on time scales via the notion of the diamond-alpha derivative. These inequalities are related to the function $f^{k}$.

\section{Acknowledgements}

The author would like to thank the anonymous referees for their constructive comments.

\section{Competing interests}

The author declares that he has no competing interests. The author states that no funding source or sponsor has participated in the realization of this work. 
Author's contributions

The work as a whole is a contribution of the author.

\section{Publisher's Note}

Springer Nature remains neutral with regard to jurisdictional claims in published maps and institutional affiliations.

Received: 14 February 2017 Accepted: 30 May 2017 Published online: 17 June 2017

\section{References}

1. Fayyaz, T, Irshad, N, Khan, A, Rahman, G, Roqia, G: Generalized integral inequalities on time scales. J. Inequal. Appl. 2016, $235(2016)$

2. Zhao, Z, Xu, B, Li, Y: Dynamic inequalities on time scales. Int. J. Pure Appl. Math. Sci. 22(1), 49-56 (2005)

3. Agarwal, R, Bohner, M, Peterson, A: Inequalities on time scales: a survey. Math. Inequal. Appl. 4, 535-557 (2001)

4. Atasever, N, Kaymakçalan, B, Lešaja, G, Taş, K: Generalized diamond- $\alpha$ dynamic Opial inequalities. Adv. Differ. Equ. 2012, 109 (2012)

5. Agarwal, R, Bohner, M: Basic calculus on time scales and some of its applications. Results Math. 35(1-2), 3-22 (1999)

6. Agarwal, R, Bohner, M, O'Regan, D, Peterson, A: Dynamic equations on time scales: a survey. J. Comput. Appl. Math. $141,1-26(2002)$

7. Bohner, M, Kaymakçalan, B: Opial inequalities on time scales. Ann. Pol. Math. 77(1), 11-20 (2001)

8. Kaymakçalan, B, Ozkan, U: Basics of diamond- $\alpha$ partial dynamic calculus on time scales. Math. Comput. Model. 50(9-10), 1258-1261 (2009)

9. Agarwal, R, Pang, P: Opial-type inequalities involving higher order derivatives. J. Math. Anal. Appl. 189(1), 85-103 (1995)

10. Bohner, M, Mahmoud, R, Saker, S: Discrete, continuous, delta, nabla and diamond-alpha Opial inequalities. Math. Inequal. Appl. 18(3), 923-940 (2015)

\section{Submit your manuscript to a SpringerOpen ${ }^{\circ}$} journal and benefit from:

- Convenient online submission

Rigorous peer review

Open access: articles freely available online

High visibility within the field

Retaining the copyright to your article

Submit your next manuscript at $\boldsymbol{~ s p r i n g e r o p e n . c o m ~}$ 